\title{
Flow Characteristic of Staggered Multiple Slotted Tubes in the Passage of a Fin Tube Heat Exchanger
}

\author{
Sedat Yayla* \\ Yuzuncu Yil University, Department of Mechanical Engineering, Turkey
}

The flow characteristics of inline and staggered cylinders placed in a fin tube heat exchanger model are experimentally investigated for Reynolds numbers of 1500 and 4000, having a duct height to cylinder diameter ratio of 0.4. In this study, the Reynolds number is calculated based on the cylinder diameter. Particle image velocimetry (PIV) is studied to obtain instantaneous representations of the non-steady flow for a specified flow field. Time-averaged flow data clearly indicates that flow through semi-cylinders has a substantial effect on the turbulent flow characteristics. A variation of time-averaged flow data along a specific line is also presented graphically. The flow interactions in the streamwise and cross-stream bases of the staggered cylinders with a flat plate surface result in a three-dimensional and a complex flow behaviour.

Keywords: particle image velocimetry (PIV), tube heat exchanger, turbulent flow, staggered slotted cylinders

\section{O INTRODUCTION}

Increasing populations and the economic, social and industrial development of countries have resulted in a huge demand for energy in the developing world; therefore, all countries throughout the world are investigating alternative energy sources. For such a need, a heat exchanger is one of the devices that can be developed further with regard to energy saving. Tube heat exchangers are commonly used in a wide variety of industrial, thermal, commercial and household applications, such as in air conditioning systems, unit heaters and radiators in condensers and evaporators.

The heat transfer problem and the efficiency of the heat exchanger are strongly related to the flow structure. The flow structure includes developing velocity and thermal boundary layers over the tubes. In heat exchangers, as the flow approaches the tubes, a horseshoe vortex system forms on the front part of each tube in the stagnation area near the tube and the fin junction region [1]. The occurrence of horseshoe vortices introduces additional mixing of hot and cold fluids in the heat exchanger flow passage and consequently increases the fin heat transfer in that area. Horseshoe vortices around the cylinder surface and a detailed flow structure affecting the heat transfer coefficient in the heat exchanger flow passage are reported in detail by Tutar and Akkoca [2].

There are many important reviews in the literature regarding flow characteristics downstream of the circular cylinder. A detailed review of the wake vortex dynamics for the circular cylinder is given by Williamson [3]. One of the most fundamental problems in fluid mechanics is determining the flow field around the cylinder surface. Horseshoe vortices, vortex shedding, and wake regions of circular cylinders at moderate Reynolds numbers have long been topics of interest for many fluid dynamics researchers. Improvements in theoretical developments and experimental techniques over the previous decade have created a perfect insight into the nature of the flow dynamics in the near wake of circular cylinders.

Structural vibrations and acoustic noise or resonance caused by periodic surface loading have led to an increase in the unsteady forces acting on the bluff bodies. However, unsteady behaviour of the vortex shedding downstream of the cylinder enhances the heat transfer. Flow control may be accomplished by controlling the boundary layer separation and/or the structure of shear layer(s) in the wake and various methods like blowing, suction, surface roughness elements, splitter plates, small rods, base bleed, etc., as has been studied by many researchers in the past. Passive control techniques suppress the vortex shedding by modifying the shape of the bluff body or by attaching additional devices in the flow field. Active control techniques utilise external energy to change the flow field. Since it is simpler and easier to implement passive techniques compared to active control techniques [4], these techniques have been widely used for flow control applications.

The literature survey partly summarises current research on vortex-bluff body interactions, physical aspects of vortical flow, and control of vortices. Different control techniques can be used to control vortex formation around the staggered arrangements of slotted cylinders. Investigations such as [5] to [8] use passive control techniques in their studies. Passive control of vortex shedding behind a circular cylinder in shallow water flow is studied by Akilli et al. [5]. They obtain a flow structure that is more changed by the 
splitter plate length in the wake region of the circular cylinder. In [6], there are more effects of fin spacing on four-row annular-finned tube bundles in staggered and in-line arrangements for the flow control. They report that the horseshoe vortex effect is quite clear in the largest fin spacing and at high Reynolds numbers. In another study on passive control, flow and heat transfer in a plate fins/circular tube assembly is researched using naphthalene sublimation technique [7]. They use the gap to tube diameter ratio, the Reynolds number and the tube location for a single tube for the researched parameters. They found that the horseshoe vortex formed in front of the tube yields a greater increase in the heat transfer rate. In the case of fin-tubes heat exchanger, the spiraling motion of the horseshoe vortex system results in better mixing and the heat transfer in the juncture location [8]. Prediction of flow characteristics thorough the heat exchangers passage is necessary for efficient design.

$\mathrm{Xu}$ and Zhou [9] state that the heat and momentum transfer characteristics downstream of heated cylinders are also strongly affected by the interaction of wake flow structures. Onal [10] reports on a number of specially configured tube shapes used in heat exchangers to improve the thermal performance effectively and to significantly reduce the size and weight of the heat exchangers. The shapes of these types of heat exchangers tubes are round, elliptical, flat, oval and rectangular.

Many researchers have performed experiments to investigate effects of different geometrical parameters on the heat transfer, and the performance of heat exchangers. Yataghene et al. [11] focus on experimental analysis of the flow patterns inside scraped surface heat exchanger (SSHE) under isothermal and continuous flow conditions. The velocity vectors of these two flows form a helical fluid flow pattern in the middle section of the exchanger. Their results indicate that a significant effective flow under certain experimental flow conditions of rotating velocity and axial flow rate can be attained. Solano et al. [12] investigate a new type of rig, whose rotating shaft is changed by a concentric reciprocating rod. They obtain a satisfactory coherence between PIV measurements and numerical results obtained by FLUENT software. In [13], velocity vectors in a vertical coaxial double-duct heat exchanger for parallel rising flow of water under conditions of laminar mixed convection have been obtained experimentally, using the PIV technique. Sahin et al. [14] report the horseshoe vortex in a rectangular duct with a narrow gap modeled to simulate a fin-tube heat exchanger containing a single circular cylinder by using the
PIV method. Variations in the size and intensity of the vortex structures with Re have been observed. Wen et al. [15] research the flow characteristics in the header of a plate-fin heat exchanger by PIV. They find that yield of fluid mal-distribution in a conventional header is highly significant while the sophisticated header configuration with a punched baffle can effectively increase the uniformity of flow distribution.

In this study, the flow control for staggered slotted cylinders in a confined flow and similar to a heat exchanger flow passage is investigated by using the PIV technique. The flow behaviour in the heat exchanger flow passage is extremely complex, three-dimensional and unsteady with flow separation, reattachment, recirculation zones, and vortices in the wake of the slotted cylinders.

\section{EXPERIMENTAL STUDY}

\subsection{Experimental Set-Up and Measurement Technique}

A water-free channel surface was used to conduct the experimental set-up. The internal dimensions of the water channel test section were $8000 \mathrm{~mm}$ long, 1000 $\mathrm{mm}$ wide and $750 \mathrm{~mm}$ high, and made of a $15 \mathrm{~mm}$ thick transparent Plexiglas sheet. The speed control unit driven by a $15 \mathrm{~kW}$ centrifugal pump was used to form the water flow. A uniform free-stream velocity distribution was provided with flow passes through a two-to-one channel contraction before reaching the test section adjusted to a $610 \mathrm{~mm}$ depth of water.

A computer, a synchroniser, a frame grabber with a maximum frame rate of $30 \mathrm{~Hz}$, a CCD camera with a resolution of $1600 \times 1186$ pixels, a double-pulsed Nd: YAG Laser source with a wavelength of $532 \mathrm{~nm}$ were part of the Dantec Dynamics PIV system. The CCD camera was placed under the water channel in the plan-view plane for the velocity measurements. The camera was compatible with the Scheimpflug condition and hence focused over the centre of the measuring plane by sensitive tilting with respect to the lens axis; $60 \mathrm{~mm}$ lenses were used in the two cameras. The Dantec Dynamics PIV system and Flow Manager Software were used to perform the measurements and to process the data. Input buffers were used to read and store the image maps. A highspeed digital frame grabber was utilised to transfer the images from the camera to the computer. With the use of a synchroniser, the correct sequence and timing triggered the laser pulses and camera. The fluid motion was observed by the water seeded with neutrally buoyant silver-coated spherical particles, 
$12 \mu \mathrm{m}$ in diameter. The output of the $120 \mathrm{~mJ} /$ pulse of maximum laser energy illuminated the measurement plane. A $1.5 \mathrm{~ms}$ time interval between pulses for all measurements was applied. The illuminating measurement plane was approximately as thick as a $1.5 \mathrm{~mm}$ laser sheet. The interrogation window was obtained according to the time interval, and the laser sheet's thickness was selected when the maximum amount of particles were displaced. The CCD camera was used to record the movement of the particles. The row displacement vector was calculated by using frame-to-frame adaptive correlation technique. The time interval between the laser pulses was used to determine the raw velocity vector field from this displacement vector field. Spurious velocity vectors (less than 5\%) were removed using the local medianfilter technique and replaced by using a bilinear least square fit technique between surrounding vectors. The Gaussian smoothing technique was used to avoid dramatic changes in the velocity field for the smoothed velocity vector field. From the circulation around the eight neighbouring points, the vorticity value at each grid point was calculated. Generally, the seeding particle size, non-uniform particle distribution, particle overlap, interrogation window's size and electronic and optical image noise caused uncertainty in velocity measurements. For each continuous running, an acquisition frequency of 15 $\mathrm{Hz}$, a total of 350 instantaneous velocity vector fields were taken. In order to obtain the averaged time velocity vectors and other flow characteristics, these instantaneous flow images were captured, recorded and stored on a computer. The plan view and side view images of the full frame area are $60 \times 70 \mathrm{~mm}^{2}$ and $46 \times 20 \mathrm{~mm}^{2}$, respectively. Westerweel [16] uses a similar digital PIV technique in a study calculating the uncertainty in the velocity field with a less than $2 \%$ error. Extensive information is reported on these uncertainty factors, which affect the PIV measurements by Gallanzi [17]. Hart [18] perform a two-pass digital PIV by using the Matlab program. He presents a non-post-interrogation method of reducing sub-pixel errors and eliminating spurious vectors from PIV results. Adrian [19] mentions the material below complements expositions of established PIV methods based on image correlation.

The laser light propagates easily due to the test geometry of the cylinders and the Plexiglas plates. Cleanvec software was used to detect and remove the spurious vectors before the image processing and then the digital images were improved, and the neighbourhood averaging technique was used for

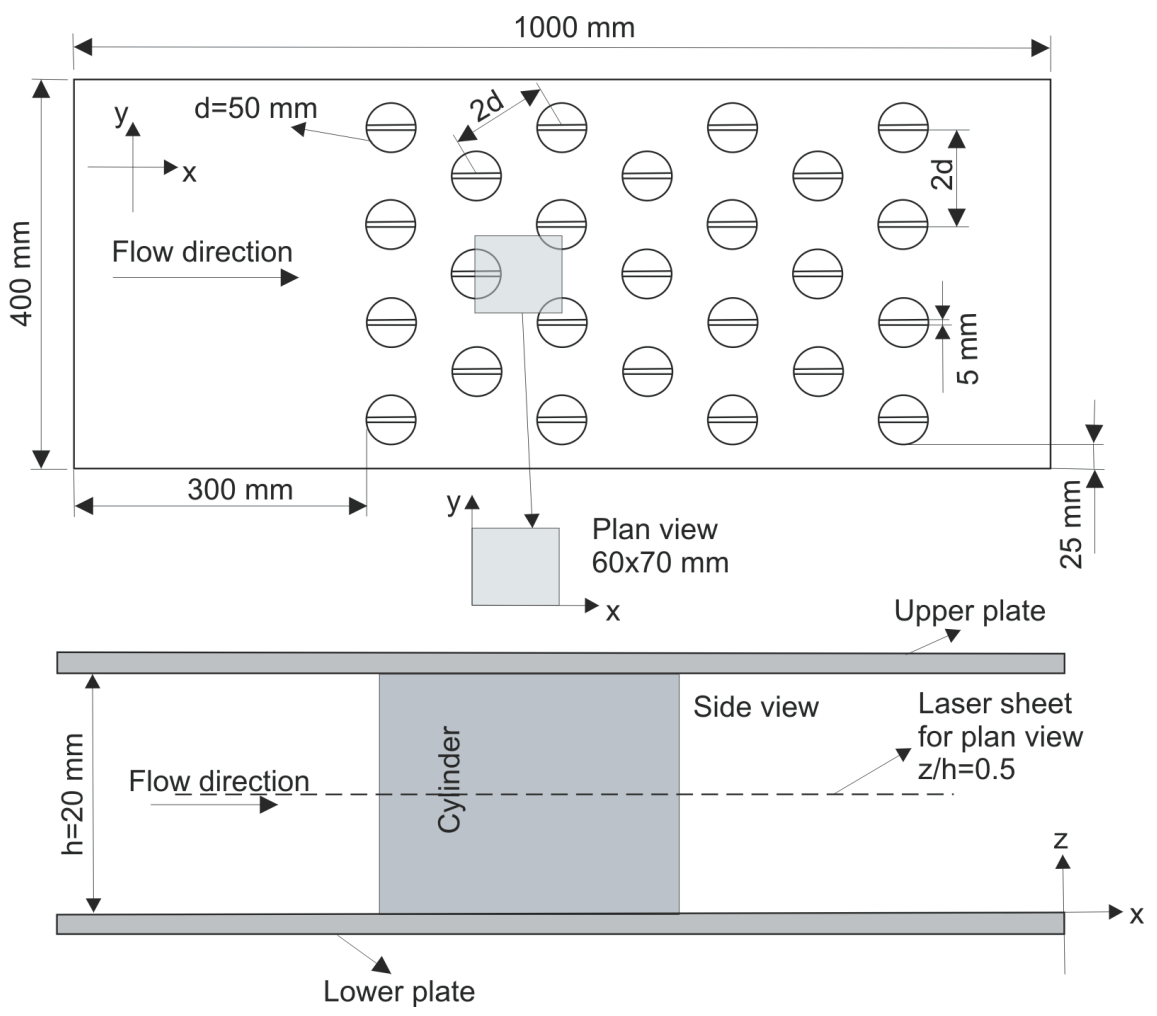

Fig. 1. Schematic representation of laser sheets, plan and side views of test section (all dimensions are in mm) 
smoothing. Fig. 1 shows the schematic of multiple staggered slotted cylinders in a narrow gapped rectangular duct used in the present experiment.

The ratio of the laser sheet height to water height was 0.5 , and it was used to determine the velocity field around the surface mounted cylinder in the passage by the PIV technique in this study. Flow between semi-cylinders was allowed by cutting the cylinder in to two pieces with a $5 \mathrm{~mm}$ whole cross-section slot. The diameter and the height of the cylinder were 50 to $20 \mathrm{~mm}$. The $R e_{d} 1500$ and 4000 are the Reynolds numbers based on the cylinder diameter.

\subsection{Experimental Results and Discussion}

Understanding the flow characteristics for the staggered cylinders placed in a fin tube heat exchanger flow passage is significant in terms of design and efficiency. Velocity vector maps, patterns of streamline, vorticity contours, Reynolds stress contours and turbulent kinetic energy, spectral analysis and the root mean square of the velocity components are presented to reveal the details of the flow. Timeaveraged flow data are obtained by averaging of 350 PIV images.

Fig. 2 illustrates the distributions of stream-wise velocities, $\langle u / U\rangle$, and the time-averaged normalised Reynolds stress correlations, $\left\langle u^{\prime} v^{\prime}\right\rangle / U^{2}$, and time- averaged vorticity contours, $\langle\omega\rangle$, for $R e_{d}=1500$ and 4000 across the flow passage. The minimum and incremental values are $[\langle u\rangle / U]_{\min }=0.05$, and $\Delta[\langle u\rangle / U]=0.05$. The distributions of stream-wise velocities, $\langle u / U>$ presented in Fig. 2 clearly indicate that the wake flow area is extremely small. This result reveals that the wake flow region has a velocity distribution with high magnitude. A pair of vortices with an opposite sign appears as seen in the last row of Fig. 2. In the field of time-averaged vorticity contours, $<\omega>$ patterns of negative vorticity are indicated with dashed lines, while positive vorticity is indicated by solid lines. Positive signed vortices or counter-clockwise rotating vortices occur along the slotted cylinder. As shown in Fig. 2, both negative and positive vorticity layers are observed along the flat plate for $R e_{d}=1500$ and 4000. Minimum and incremental values of the vorticity are $\omega_{\min }= \pm 1 \mathrm{~s}^{-1}$ and $\Delta \omega=2 \mathrm{~s}^{-1}$. Stronger vorticity layers are obtained for higher Reynolds numbers. Time-averaged normalised Reynolds stress correlations, $u^{\prime} v^{\prime} / U^{2}$, in the downstream vicinity of the slotted cylinders were observed as shown in the second row of Fig. 2. The minimum and incremental values of normalised Reynolds-stress are $\left[\left\langle u^{\prime} v^{\prime}\right\rangle / U^{2}\right]$ $\min = \pm 0.002$ and $\Delta\left[\left\langle u^{\prime} v^{\prime}\right\rangle / U^{2}\right]=0.002$, respectively. The negative and the positive normalised Reynolds stress contours are $\left\langle u^{\prime} v^{\prime}\right\rangle / U^{2}$ represented with dashed and solid lines, respectively. It can be concluded that,
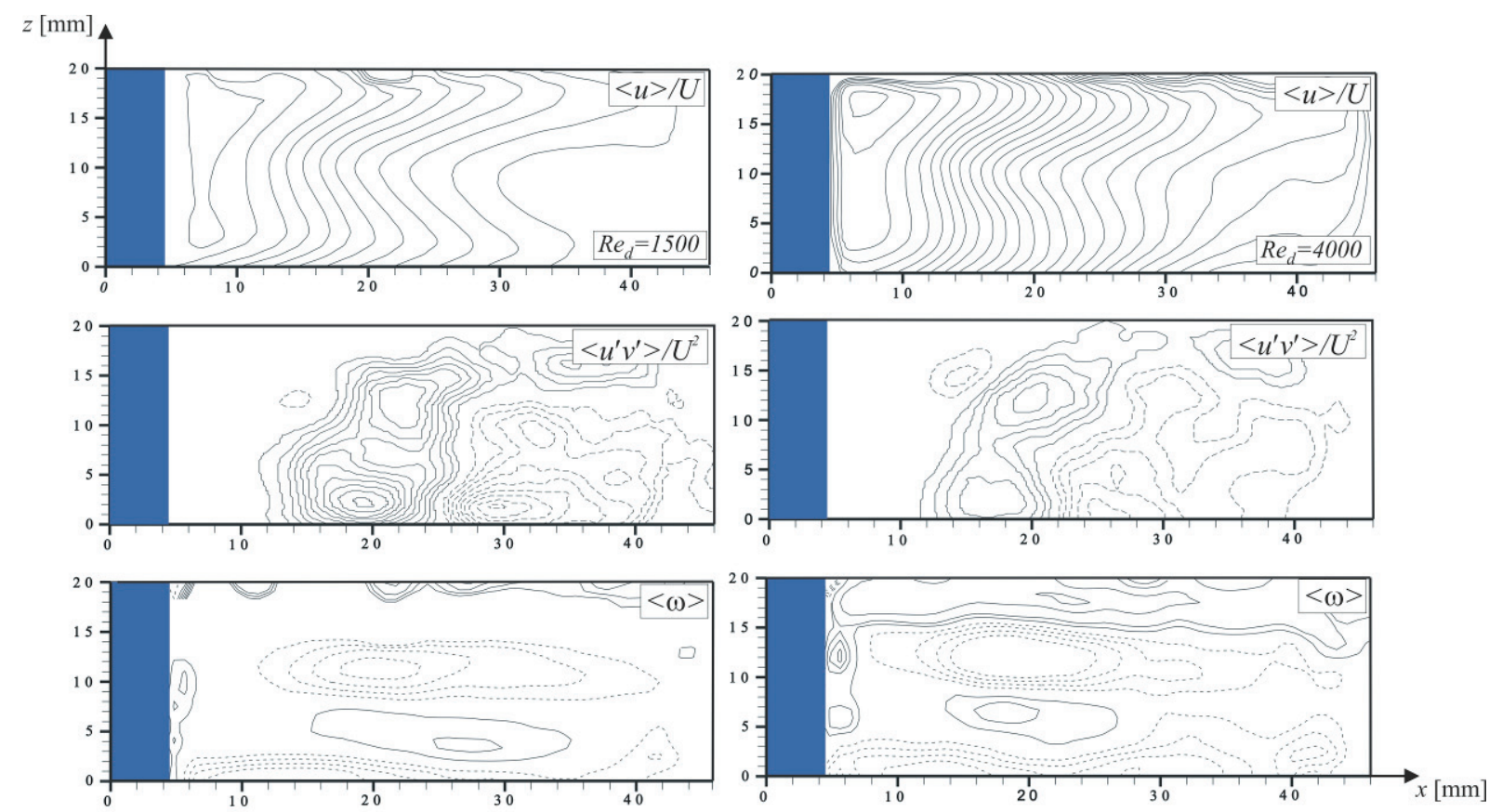

Fig. 2. Pattern of time-averaged component of stream-wise velocity, $[\langle u\rangle / U]$ normalised Reynolds stress contours, $\left[\left\langle u^{\prime} v^{\prime}\right\rangle / U^{2}\right]$ and vorticity contours, $\langle\omega\rangle$ in the downstream base of the cylinder in side-view planes for Red $=1500$ and 4000 

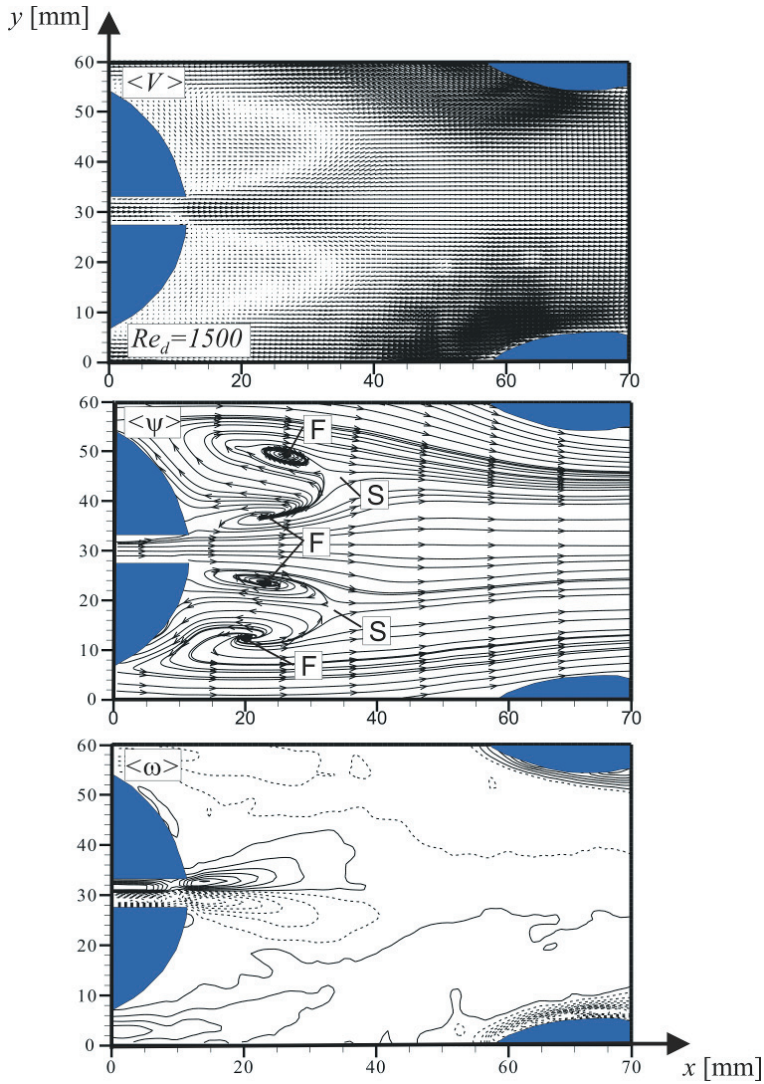

Fig. 3. Time-averaged velocity vector field $\langle V\rangle$, corresponding streamline topology $\langle\psi\rangle$ and vorticity contours $\langle\omega\rangle$ for $R e_{d}=1500$; minimum and incremental value of vorticity are $\omega_{\min }= \pm 1 \mathrm{~s}^{-1}$ and $\Delta \omega=2 \mathrm{~s}^{-1}$

downstream of the slotted cylinder, the peak values of the normalised Reynolds stress correlations, $\left\langle u^{\prime} v^{\prime}\right\rangle / U^{2}$ decrease as the Reynolds number increases.

Flow characteristics downstream of the slotted cylinders are more important in terms of the heat transfer rate in comparison to the forward faces of slotted cylinders, since the occurrence of the wake flow region hydro-dynamically deteriorates the rate of heat transfer. Thus, more attention must be paid for these regions. The time-averaged flow data, which includes the velocity vector map, $\langle V\rangle$, the patterns of streamlines, $\langle\psi\rangle$ and the contours of vorticity, $\langle\omega\rangle$ at an elevation of $z / h=0.5$, are presented in Fig. 3 for Reynolds numbers $R e_{d}=1500$. As seen in the image, three cylinders were included in one frame of an image in order to see the cylinder and flow interactions. In the first frame of images, the averaged velocity vector map $\langle V\rangle$ indicates that a high rate of velocity occurs on the shoulders of each cylinder. Patterns of time-averaged components of the velocity vector, $\langle V\rangle$, indicate that vortex formation occurs immediately adjacent to the

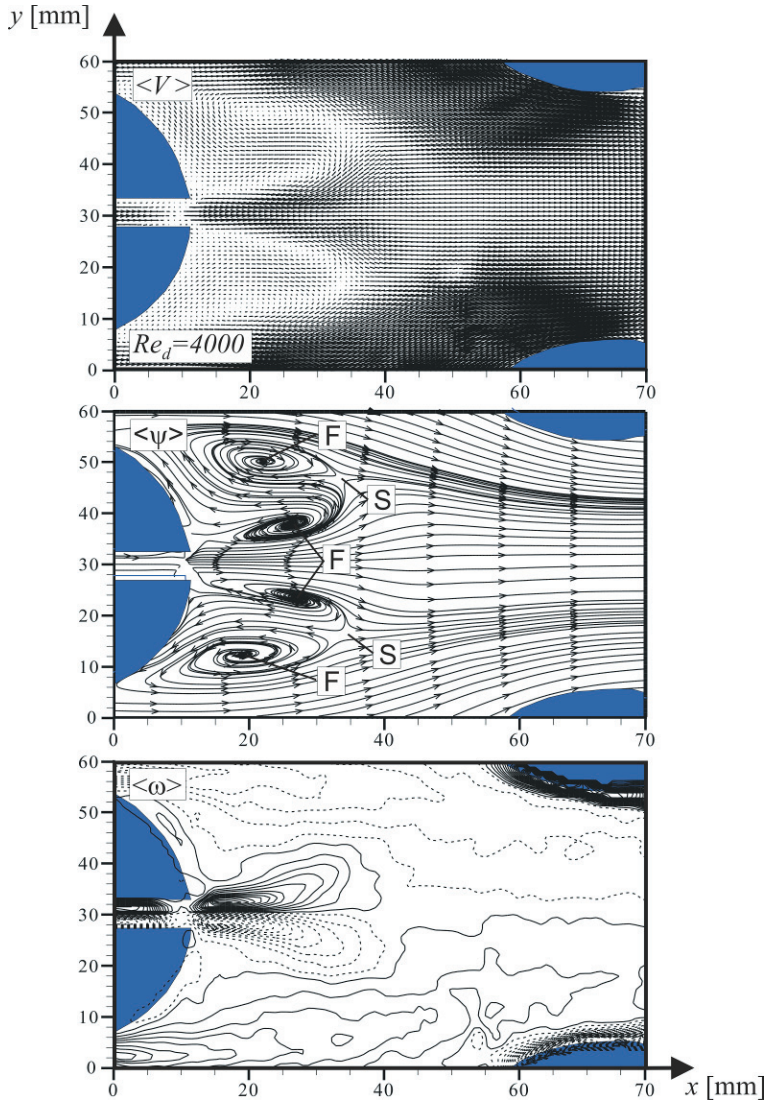

Fig. 4. Time-averaged velocity vector field $\langle V\rangle$, corresponding streamline topology $\langle\psi\rangle$ and vorticity contours $\langle\omega\rangle$ for $R e_{d}=4000$; minimum and incremental value of vorticity are $\omega_{\min }= \pm 1 \mathrm{~s}^{-1}$ and $\Delta \omega=2 \mathrm{~s}^{-1}$

base of the cylinder and as these horseshoe vortices immigrate in the direction of the free-stream flow; they also flap in the lateral direction in an unsteady fashion. In the second line of the images, the time-averaged streamline patterns, $\langle\psi\rangle$, show that separated flow regions are more compact, since the water jet flow goes through slots directly into the wake flow region. Streamline patterns, $\langle\psi\rangle$, illustrate that fresh flow occurred due to the hydrodynamics of the horseshoe vortex system and from the slot is transported in to the wake flow regions for further extent. The corresponding streamline patterns reveal that symmetrical foci with opposite circulation and saddle points downstream are evident. The centres of the foci and the saddle point are designated as $F$ and $S$, respectively, as indicated in Fig. 3. There is a saddle point, $S$, for each focus, $F$. In the last line of the images of the time-averaged vorticity contour, the $\langle\omega\rangle$ values reveal that the present shear layers emerge from both sides of the slotted cylinders moving downstream in between neighbouring slotted cylinders and interact with the shear layers of other 
cylinders for $\mathrm{z} / \mathrm{h}=0.5$. In Fig. 3, a symmetrically distributed cluster of positive (solid line) and negative (dashed line) vorticity layers beginning around the inlet region of slots evidently extends further downstream around the plane of symmetry of the slotted cylinder. Two pairs of strong positive and negative vorticity of horseshoe vortex systems are detected adjacent to the outer region of the shear layer. It seems that the water jet flow sucks the horseshoe vortex in a way that the primary horseshoe vortex comes extremely close to the inlet region of slots.

Images for downstream flow of time-averaged data of the slotted cylinders at an elevation of $z / h=$ 0.5 for Reynolds numbers $R e_{d}=4000$ are presented in Fig. 4. As it is clearly seen, locations of foci are more or less the same as presented by the time-averaged velocity vector, $\langle V\rangle$, distributions and corresponding patterns of streamlines, $\langle\psi\rangle$, as the Reynolds number is increased from 1500 to 4000 . The structure of the flow changes as a function the Reynolds number. Wake flow regions in lateral directions thicken slightly with the Reynolds number due to the presence of the other slotted cylinders. In the last line of the images, more compact, stronger, and symmetrically distributed positive and negative vorticity clusters beginning from the vicinity of the slot in the slottedcylinder surface and convecting further downstream around the plane of symmetry are observed.

In addition to time-averaged and instantaneous flow data, phase-averaged data also provide detailed information on the structure of the flow field. The history of development of the flow structure is computed using a sequence of 350 instantaneous images of velocity vectors. The natural frequency of shedding vortices is obtained using Fast Fourier Transformation (FFT) analysis and natural frequency spectrum. The spectra of stream-wise velocity fluctuations obtained using FFT analysis for four selected points in the vertical symmetry plane are presented in Fig. 5. The location of the selected points are near the slot $(x / d=0.36, y / d=0.05)$ and $(x / d=$ $0.36, y / d=1.12)$, far the slot $(x / d=0.36, y / d=0.54)$ and $(x / d=0.36, y / d=0.64)$ in the wake region. The dominant frequency of $f=4.8 \mathrm{~Hz}$ at four points is
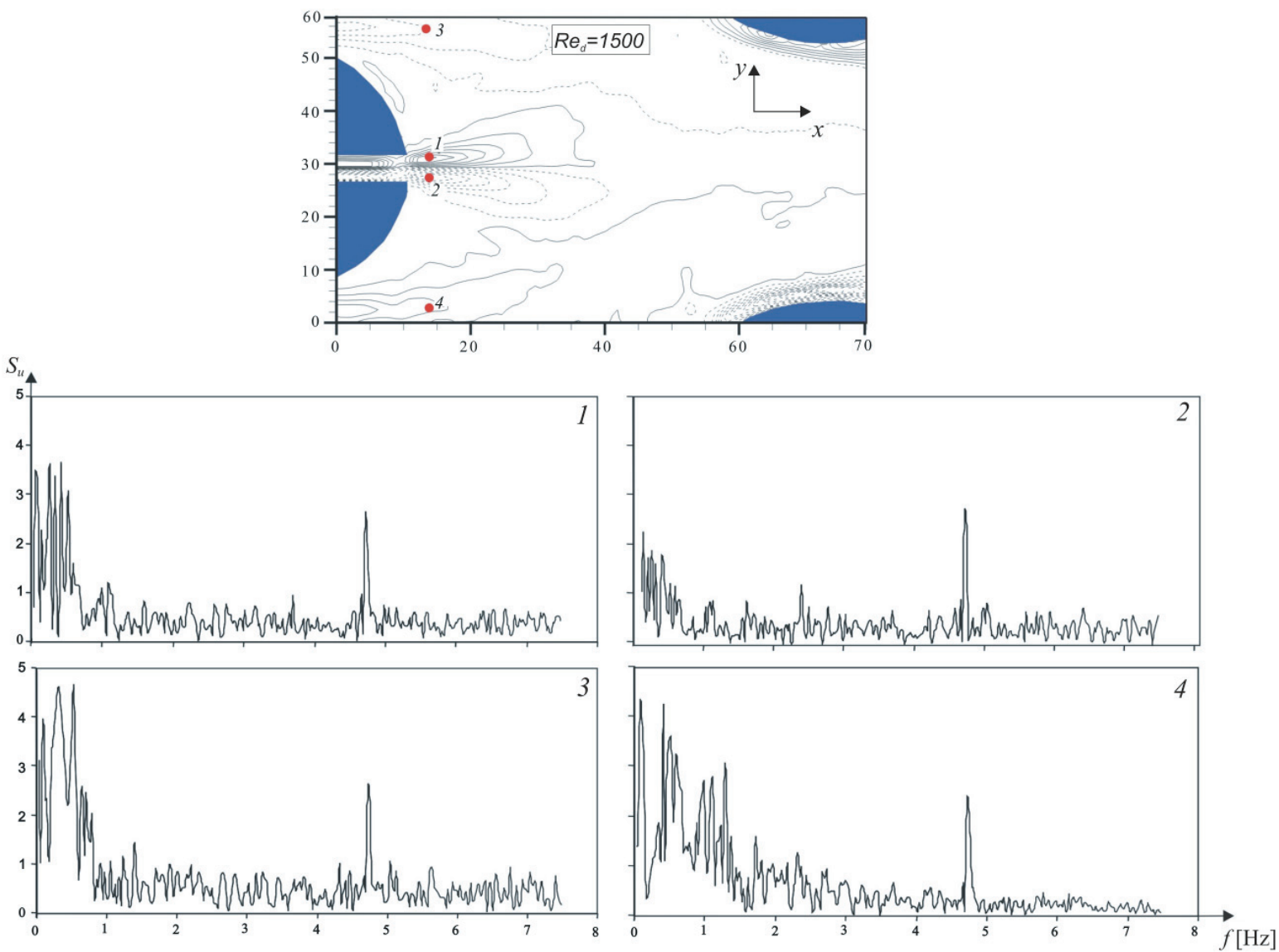

Fig. 5. Comparison of the power spectra calculated at various locations of the flow field for $\operatorname{Re}_{d}=1500$ 
close to the slotted cylinder for the Reynolds number of $R e_{d}=1500$.

The distance from the slot of the point where the dominant frequency occurs is not the same for Reynolds numbers 1500 and 4000 as indicated in Fig. 6 . The dominant frequency in the location of selected points $(x / d=0.36, y / d=0.54)$ and $(x / d=0.36, y / d=$ $0.64)$ is $f=0.81 \mathrm{~Hz}$ and the other two points $(x / d=$ $0.36, y / d=0.05)$ and $(x / d=0.36, y / d=1.12)$ is $f=1.33$ $\mathrm{Hz}$ for the Reynolds number $R e_{d}=4000$. However, the spectra taken locations of near the slot (points 1 and 2) shows a clear and distinct peak at $f=0.81$, which is smaller than the natural vortex shedding frequency (points 3 and 4) obtained from the slotted-cylinder case.

Fig. 7 illustrates time-averaged normalised Reynolds stress correlations, $\left\langle u^{\prime} v^{\prime}\right\rangle / U^{2}$, distributions stream-wise and transverse velocities, $u / U$ and $v / U$, for $R e_{d}=1500$ and 4000 across the flow passage in a vertical plane at the central cross-sections downstream of the slotted cylinder. The minimum and the incremental values of each variable are indicated in the images. In each image, contours of positive velocity components are indicated by solid lines and dashed lines indicate negative velocity components. In the first line of the images, the high levels of negative pockets of time-averaged stream-wise velocity contours, $\langle u\rangle / U$, occur in plain view further downstream as the Reynolds number is increased. A cross comparison of the images shown in the mid-line of Fig. 7 with corresponding patterns of time-averaged streamline shown in Figs. 4 and 3 indicate that concentration of $\langle v\rangle / U$ occurs in between two foci $F$ and saddle point $S$. The corresponding normalised Reynolds stress contours $\left(\left\langle u^{\prime} v^{\prime}\right\rangle / U^{2}\right.$, where $u^{\prime}$ and $v^{\prime}$ are fluctuations of $u$ and $v$, respectively) are shown in the last line of the images. The peak values of positive and negative Reynolds stress contours indicate the regions under high fluctuations. However, the peak values of Reynolds stress correlation, $\left\langle u^{\prime} v^{\prime} / U^{2}\right\rangle$ occurs along the shear layer. It is clear that for $R e_{d}$ $=4000$, the momentum of the jet flow has a fairly large magnitude and therefore, locally higher values

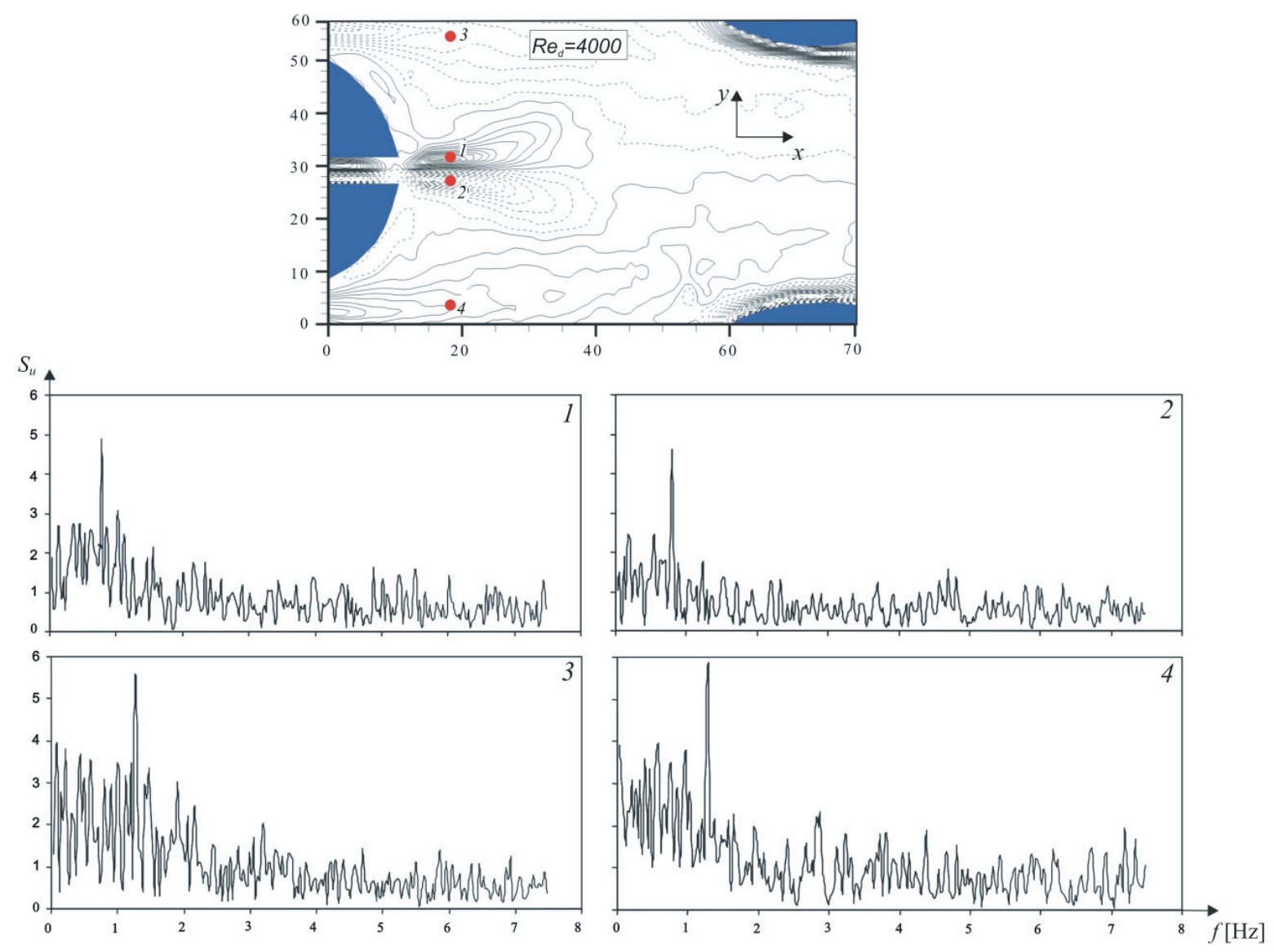

Fig. 6. Comparison of the power spectra calculated at various locations of the flow field for $\operatorname{Re}_{d}=4000$ 

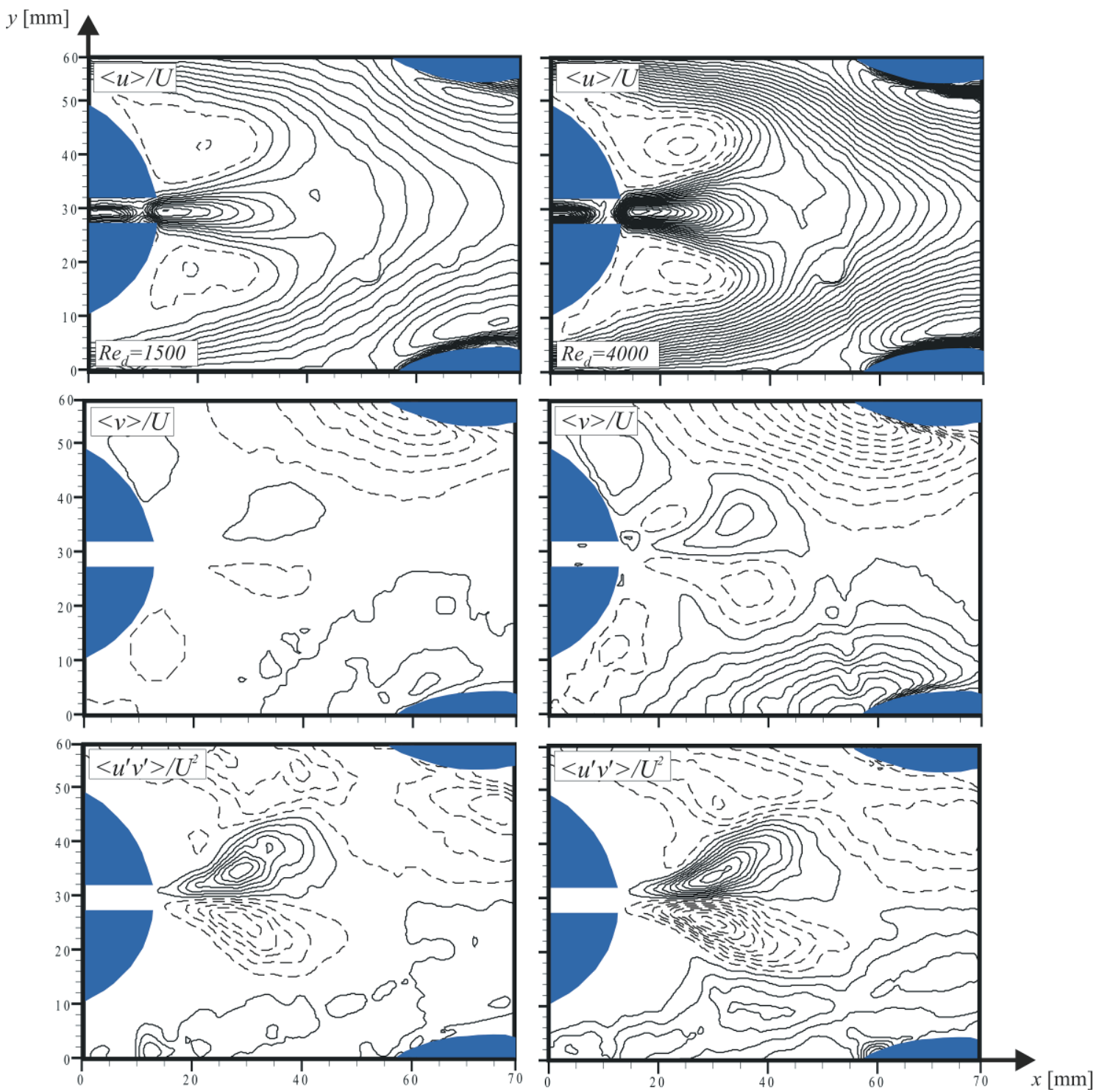

Fig. 7. Patterns of time-averaged components of stream-wise, $[\langle u\rangle / U]$ transverse, $[\langle v\rangle / U]$ velocity, normalised Reynolds stress contours, $\left[\left\langle u^{\prime} v^{\prime}\right\rangle / U^{2}\right]$ and for $R e_{d}=1500$ and 4000; minimum and incremental values are $[\langle u\rangle / U]_{\min }=0.025,[\langle v\rangle / U]_{\min }=0.05$ and $\Delta[\langle u\rangle / U]=0.025, \Delta[\langle v\rangle / U]=0.05$; for contours of normalised Reynolds-stress, $\left[\left\langle u^{\prime} v^{\prime}\right\rangle / U^{2}\right]_{\min }= \pm 0.01$, and $\Delta\left[\left\langle u^{\prime} v^{\prime}\right\rangle / U^{2}\right]=0.02$

of $\left\langle u v^{\prime} / U^{2}\right\rangle$ that are obtainable in the region of jet formation.

The turbulent flow characteristics are provided in terms of root mean square values of stream-wise velocity, $u,\left(u_{r m s}\right)$ and transverse velocity, $v,\left(v_{r m s}\right)$ in order to identify regions of high fluctuations as shown in Fig. 8. Both $u_{r m s}$ and $v_{r m s}$ are normalised by free stream velocity, $U$, indicating the peak value of these $r m s$ of velocity components gradually increasing as the Reynolds number in between neighbouring slotted cylinders is increased. The location of the maximum value of $\left\langle u_{r m s}\right\rangle / U$ is kept in the same distance from the slotted-cylinder surface. Contours of $v_{r m s} / U$ are stacked far downstream of the cylinder as well as in the proximity of the upper surface of the cylinder at both sides as seen in the last line of Fig. 8. Peak values of $u_{r m s} / U$ for Reynolds numbers of 1500 and 4000 are 0.25 and 0.0 .35 , and also $v_{r m s} / U$ are 0.1 and 0.2 , respectively. The minimum and incremental values of normalised root mean square of stream-wise and transverse velocity fluctuations are given in the figure captions.

The results of the turbulent kinetic energy (TKE) for Reynolds numbers of 1500 and 4000 are shown in Fig. 9. The minimum and incremental values of the contours of time-averaged turbulent kinetic 


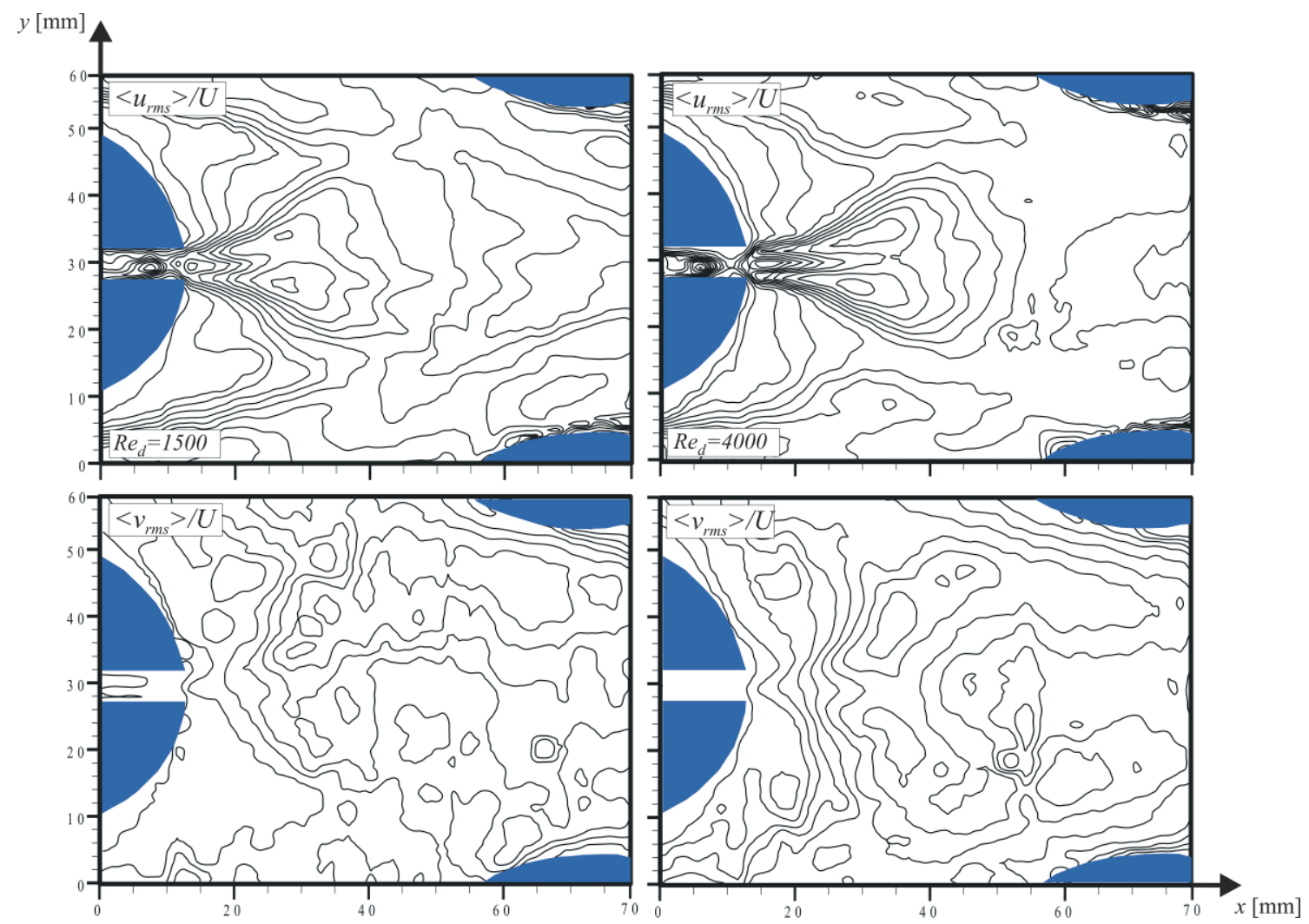

Fig. 8. Patterns of rms of the stream-wise, transverse velocity fluctuation respectively, $u_{r m s} / U, v_{r m s} / U$ for $R e_{d}=1500$ and 4000 ; minimum and incremental values are $\left[u_{r m s} / U\right]_{\min }=0.05,\left[v_{r m s} / U\right]_{\min }=0.05$ and $\Delta\left[u_{r m s} / U\right]=0.05, \Delta\left[v_{r m s} / U\right]=0.05$
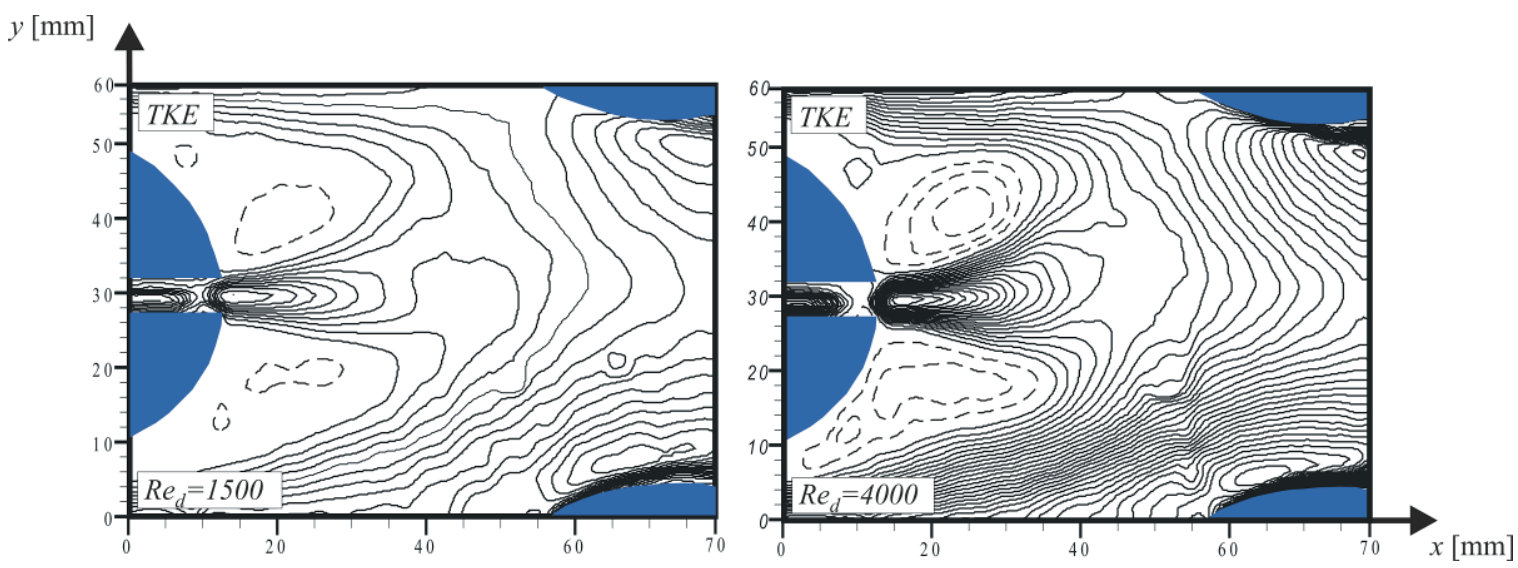

Fig. 9. Turbulent kinetic energy (TKE) distribution downstream of the cylinder for $\operatorname{Re}_{d}=1500$ and 4000

energy remain same for all cases: $\left[\langle T K E>]_{\min }=3\right.$ and $\Delta[<T K E>]=4$, respectively. For the two graphs, the values of turbulent kinetic energy are extremely high close to the slot location due to the jet-like flow. However, it decreases gradually in shear layers at both sides of the slotted cylinder. The maximum value of the normalised TKE for Reynolds numbers of 1500 and 4000 are 23 and 47, respectively. The turbulent kinetic energy is related to the root mean square of the velocity components. Fig. 8 shows that when the Reynolds number is increased, magnitudes of $u_{r m s} / U$ and $v_{r m s} / U$ are lower in shear layers at both sides of the slotted cylinder than the values of these velocity components close to the slot location of the slotted cylinder. 


\section{CONCLUSIONS}

The main purpose of this study was to investigate details of flow structure in the downstream of a circular cylinder mounted on a flat surface for multiple slot-cylinders in deep water channels, using the PIV technique. Slotted cylinders are located at the corners of equilateral triangles. Owing to the denseness of this type of heat exchanger, the distance between cylinders surfaces is $1 \mathrm{~d}$. The laser sheet was located parallel to the bottom surface of the water channel at the elevation of $\mathrm{z} / \mathrm{h}=0.5$. Due to the arrangement of the slotted cylinders, the flow structure in a plate fin tube the heat exchanger of the thermal unit is composed of staggered cylinders. In closer parts of the slotted cylinders, horseshoe vortices occurred on both surfaces of the plate. The entrainment process and circulatory motions between the core and the wake flow regions, downstream of the slotted cylinder mounted on the flat plate surface in deep water flow are magnified by these vortices. The absence of biased flow was achieved by the jet flow. Wake-flow regions of the slotted cylinders elongate in the stream-wise direction due to the momentum transfer from the slot of the cylinders. In summary, staggered cylinder arrangements enhance the heat transfer rate hydrodynamically even in wake flow regions.

\section{ACKNOWLEDGMENTS}

The author acknowledges the financial support of the BAPB (Directory of Scientific Research Projects of Yuzuncu Yil University, Turkey) for funding under project No: 2012-MIM-B012. Experimental part of the project was carried out in the Fluid Mechanics Laboratory of Mechanical Engineering Department at Cukurova University in Turkey.

\section{NOMENCLATURE}

$d \quad$ cylinder diameter

$R e_{d} \quad$ Reynolds number is calculated based on the cylinder diameter

$u \quad$ stream-wise velocity component

$v \quad$ transverse velocity component

$u^{\prime} v^{\prime} \quad$ Reynolds stress

$u^{\prime} \quad$ fluctuations of $\mathrm{u}$

$v^{\prime} \quad$ mean fluctuations of $\mathrm{v}$

$u_{r m s} \quad$ root mean square of $\mathrm{u}$

$v_{r m s} \quad$ root mean square of $\mathrm{v}$

$U \quad$ free stream velocity

$V \quad$ velocity vector $\psi \quad$ streamline

$\omega \quad$ vorticity

TKE Turbulent Kinetic Energy

$<>\quad$ time-averaged value of quantity

\section{REFERENCES}

[1] Sahin, B., Akkoca, A., Ozturk, N.A., Akilli, H. (2006). Investigation of flow characteristics in a plate fin and tube heat exchanger model composed of single cylinder. International Journal of Heat and Fluid Flow, vol. 27, no. 3, p. 522-530, DOI:10.1016/j. ijheatfluidflow.2005.11.005.

[2] Tutar, M., Akkoca, A. (2004). Numerical analysis of fluid flow and heat transfer characteristics in three dimensional plate fin and tube heat exchangers. Numerical Heat Transfer, vol. 46, no. 3, p. 1-21, DOI:10.1080/10407780490474762.

[3] Williamson, C.H.K. (1996). Vortex dynamics in the cylinder wake. Annual Review of Fluid Mechanics, vol. $28, \quad$ p. 477-526, DOI:10.1146/annurev. fl.28.010196.002401.

[4] Lee, S.J., Lee, S.I., Park, C.W. (2004). Reducing the drag on a circular cylinder by upstream installation of a small control rod. Fluid Dynamics Research, vol. 34, no. 4, p. 233-250, DOI:10.1016/j.fluiddyn.2004.01.001.

[5] Akilli, H., Karakus, C., Akar, A., Sahin, B., Tumen, N.F. (2008). Control of vortex shedding of circular cylinder in shallow water flow using an attached splitter plate. Journal of Fluids Engineering, vol. 130, no. 3, p. 1-11, DOI: $10.1115 / 1.2903813$, DOI:10.1115/1.2903813.

[6] Mon, M.S.R., Gross, U. (2004). Numerical study of finspacing effects in annular finned tube heat exchangers. International Journal of Heat and Mass Transfer, vol. 47, no. 8-9, p. 1953-1964, DOI:10.1016/j. ijheatmasstransfer.2003.09.034.

[7] Kim, J.Y., Song, T.H. (2002). Microscopic phenomena and macroscopic evaluation of heat transfer from plate fins/circular tube assembly using naphthalene sublimation technique. International Journal of Heat and Mass Transfer, vol. 45, no. 16, p. 3397-3404, DOI:10.1016/S0017-9310(02)00047-9.

[8] Tiwari, S., Biswas, G., Prasad, P.L.N., Basu, S. (2003). Numerical prediction of flow and heat transfer in a rectangular channel with a built-in circular tube. Journal of Heat Transfer, vol. 125, no. 3, p. 413-421, DOI:10.1115/1.1571087.

[9] Xu, G., Zhou, Y. (2004). Strouhal numbers in the wake of two inline cylinders. Experiments in Fluids, vol. 37, no. 2 , p. 248-256, DOI:10.1007/s00348-004-0808-0.

[10] Onal, O. (2010). Investigation of flow characteristics around a Single and staggered slotted-cylinders. Ph.D. thesis, Cukurova University, Adana.

[11] Yataghene, M., Francine, F., Jack, L. (2011). Flow patterns analysis using experimental piv technique inside scraped surface heat exchanger in continuous flow condition. Applied Thermal Engineering, 
vol. 31, no. 14-15, p. 2855-2868, DOI:10.1016/j. applthermaleng.2011.05.011.

[12] Solano, J.P., García, A., Vicente, P.G., Viedma, A. (2011). Flow pattern assessment in tubes of reciprocating scraped surface heat exchangers. International Journal of Thermal Sciences. vol. 50, no. 5, p. 803-815, DOI:10.1016/j.ijthermalsci.2010.11.019.

[13] Maré, T., Galanis, G., Voicu, L., Miriel, J., Sow, O. (2008). Experimental and numerical study of mixed convection with flow reversal in coaxial double-duct heat exchangers. Experimental Thermal and Fluid Science, vol. 32, no. 5, p. 1096-1104, DOI:10.1016/j. expthermflusci.2008.01.002.

[14] Sahin, B., Ozturk, N.A., Gurlek, C. (2008). Horseshoe vortex studies in the passage of a model plate-fin-andtube heat exchanger. International Journal of Heat and Fluid Flow, vol. 29, no. 1, p. 340-351, DOI:10.1016/j. ijheatfluidflow.2007.06.005.
[15] Wen, J., Li, Y., Wang, S., Zhou, A. (2007). Experimental investigation of header configuration improvement in plate-fin heat exchanger. Applied Thermal Engineering, vol. 27, no. 11-12, p. 1761-1770, DOI:10.1016/j.applthermaleng.2007.01.004.

[16] Westerweel, J. (1993). Digital Particle Image Velocimetry, Theory and Application. Delft University Press, Delft.

[17] Gallanzi, M.F. (1998). High accuracy measurement of unsteady flows using digital particle image velocimetry. Optics \& Laser Technology, vol. 30, no. 6-7, p. 349359, DOI:10.1016/S0030-3992(98)00020-6

[18] Hart, D.P. (1993). PIV error correction. Experiments in Fluids, vol. 29, no. 13-22, p. 13-22.

[19] Adrian, R.J. (2005). Twenty Years of Particle Image Velocimetry. Experiments in Fluids, vol. 39, no. 1, p. 159-169, DOI:10.1007/s00348-005-0991-7. 